\title{
Proton Pump Interaction
}

National Cancer Institute

\section{Source}

National Cancer Institute. Proton Pump Interaction. NCI Thesaurus. Code C40535.

Proton Pump Interaction consists of temporary non-covalent binding of a molecule

through intermolecular physical forces of attraction with a membrane associated enzyme, or complex, involved in ATP-dependent transmembrane transport of protons against a concentration gradient, often in exchange for other cations, resulting directly from the expenditure of energy. 